\title{
Scaling at the Energy-driven Metal-Insulator Transition and the Thermoelectric Power
}

\author{
Alexander Croy ${ }^{* 12}$ and Rudolf A. Römer ${ }^{2}$ \\ ${ }^{1}$ Institut für Physik, Technische Universität, 09107 Chemnitz, Germany \\ ${ }^{2}$ Physics Department \& Centre for Scientific Computing, University of Warwick, Coventry CV4 7AL, U.K.
}

Received 21 August 2005, revised 27 August 2005

Published online Revision : 1.22, compiled 15/3/2021

Key words Anderson localisation, one-parameter scaling, energy transition, thermopower PACS 72.15.Rn, 72.15.Jf, 72.20.Ee

The electronic properties of disordered systems at the Anderson metal-insulator transition (MIT) have been the subject of intense study for several decades. Thermoelectric properties at the MIT, such as thermopower and thermal conductivity, however, have been relatively neglected. Using the recursive Green's function method and the Chester-Thellung-Kubo-Greenwood formalism, we calculate numerically the low temperature behaviour of all kinetic coefficients $L_{i j}$. From these we can deduce for example the electrical conductivity $\sigma$ and the thermopower $S$ at finite temperatures. Here we present results for the case of completely coherent transport in cubic $3 \mathrm{D}$ systems.

Copyright line will be provided by the publisher

1 Introduction The Anderson model [1] is widely used to investigate the phenomenon of localisation in disordered materials. Especially the possibility of a quantum phase transition driven by disorder from an insulating phase, where all states are localised, to a metallic phase with extended states has lead to extensive analytical and numerical investigations of the critical properties of this metal-insulator transition (MIT) [2].

The one-parameter scaling theory plays a crucial role in understanding the MIT [3]. It is based on an ansatz interpolating between metallic and insulating regimes [4]. So far, scaling has been demonstrated to an astonishing degree by numerical studies of the Anderson model [5]. However, most studies focused on scaling of the localisation length and the conductance at the disorder-driven MIT in the vicinity of the band centre.

In the present paper, we will show numerical results for cubes of volume $L^{3}$ which are consistent with scaling also at the energy-driven transition across the mobility edge $E_{\mathrm{c}}$ near the band tails. In particular, we find that at $T=0$ the d.c. conductivity $\sigma(E)$ close to $E_{\mathrm{c}}$ is well described by the power-law [4 6]

$$
\sigma(E)=\left\{\begin{array}{cc}
\sigma_{0}\left|1-\frac{E}{E_{\mathrm{c}}}\right|^{\nu} & |E|<E_{\mathrm{c}} \\
0, & |E|>E_{\mathrm{c}}
\end{array}\right.
$$

Moreover, knowing the explicit energy and system size dependence of the conductance $g=\sigma L$ allows us to calculate the low-temperature dependence of thermoelectric properties such as thermopower, Lorenz number and thermal conductivity in the fully quantum coherent regime. For these, similarly detailed theoretical investigations of scaling at the MIT are still lacking. Here we present an approach based on the recursive Green's function technique [7].

* Corresponding author: e-mail: a.croy@warwick.ac.uk, Phone: +442476574 309, Fax: +44 7876858246 
2 Thermoelectric Transport Properties Thermoelectric transport properties describe the response of a material to the presence of a small external electric field and a small temperature gradient $\nabla T$. E.g., the induced (thermo-)electric field $\boldsymbol{E}$ in the Seebeck effect is directly proportional to $\boldsymbol{\nabla} T$ and the constant of proportionality is called thermopower $S$. In general, the dependency of the electric $\left(j_{\mathrm{e}}\right)$ and thermal currents $\left(j_{\mathrm{q}}\right)$ on a electric field and a temperature gradient up to linear order are [8]

$$
\boldsymbol{j}_{e}=\frac{1}{|e|}\left(|e| L_{11} E-L_{12} \frac{\nabla T}{T}\right) \quad \text { and } \quad \boldsymbol{j}_{q}=\frac{1}{|e|}\left(|e| L_{21} E-L_{22} \frac{\nabla T}{T}\right) .
$$

Here $e$ is the electron charge. This defines the kinetic coefficients $L_{i j}(i, j=1,2)$. Additionally, for zero magnetic field, the Onsager relation states that $L_{21}=L_{12}$ [8]. Using the definitions of the thermoelectric properties [8] and Eq. [2] one can express $\sigma$ and $S$ in terms of the kinetic coefficients as

$$
\sigma=L_{11} \quad \text { and } \quad S=\frac{L_{12}}{|e| T L_{11}} .
$$

Assuming elastic scattering of independent electrons by static impurities or by lattice vibrations one can obtain the kinetic coefficients from the Chester-Thellung-Kubo-Greenwood (CTKG) formulation [9 10],

$$
L_{i j}(T)=(-1)^{i+j} \int_{-\infty}^{\infty} \sigma(E)[E-\mu(T)]^{(i+j-2)}\left[-\frac{\partial f(E, \mu, T)}{\partial E}\right] d E, \quad(i, j=1,2),
$$

where $\mu(T)$ is the chemical potential and $f(E, \mu, T)$ is the usual Fermi function.

3 Model and Numerical Method The Anderson model of localisation is based upon a tight-binding Hamiltonian in site representation

$$
\mathcal{H}=\sum_{i} \varepsilon_{i}|i\rangle\left\langle i\left|-\sum_{i \neq j} t_{i j}\right| i\right\rangle\langle j|,
$$

where $|i\rangle$ is a localised state at site $i$ and $t_{i j}=t$ are the hopping parameters, restricted as usual to nearest neighbours. The on-site potentials $\varepsilon_{i} \in[-W / 2, W / 2]$ are uniformly distributed random numbers.

For calculating the energy dependence of the two-point conductance $G_{2}=e^{2} / h g_{2}$ we use the recursive Green's function method [7]. This method is based on the Kubo-Greenwood formula for the linear response regime and non-interacting electrons [10]. In order to obtain correct results for purely elastic scattering, metallic leads were attached at both ends of the system. In this case the formalism given above is equivalent [11] to the Landauer-Büttiker formulation [12]. To get the conductance $g$ of the disordered region only, we have to subtract the contact resistance due to the leads. This gives $1 / g=1 / g_{2}-1 / N$. Here $N=N(E)$ is the number of propagating channels at the Fermi energy $E$ [13]. An additional complication arises because the number of transmitting lead-modes drops to zero outside the lead-energy band, whereas the disordered system still has states at these energies. This mismatch gives rise to a lead-induced reduction in $g$. The problem can be overcome by shifting the energy of the disordered region while keeping the Fermi energy in the leads in the lead-band centre. This is equivalent to applying a gate voltage to the disordered region and sweeping it - a technique similar to MOSFET experiments.

4 Conductance Scaling and D.C. Conductivity We set the disorder strength to $W / t=12$ and impose fixed boundary conditions in the transverse direction [14]. For each combination of Fermi energy and system size we generate an ensemble of 10000 samples (except for $L=19$ and $L=21$, where 4000 and 2000 were generated) and examine the size-dependence of the average and the typical conductance, $\langle g\rangle$ and $\langle\ln g\rangle$, respectively. We find that for $E_{\mathrm{F}} / t \leq-8.2$ the typical conductance is proportional to the 


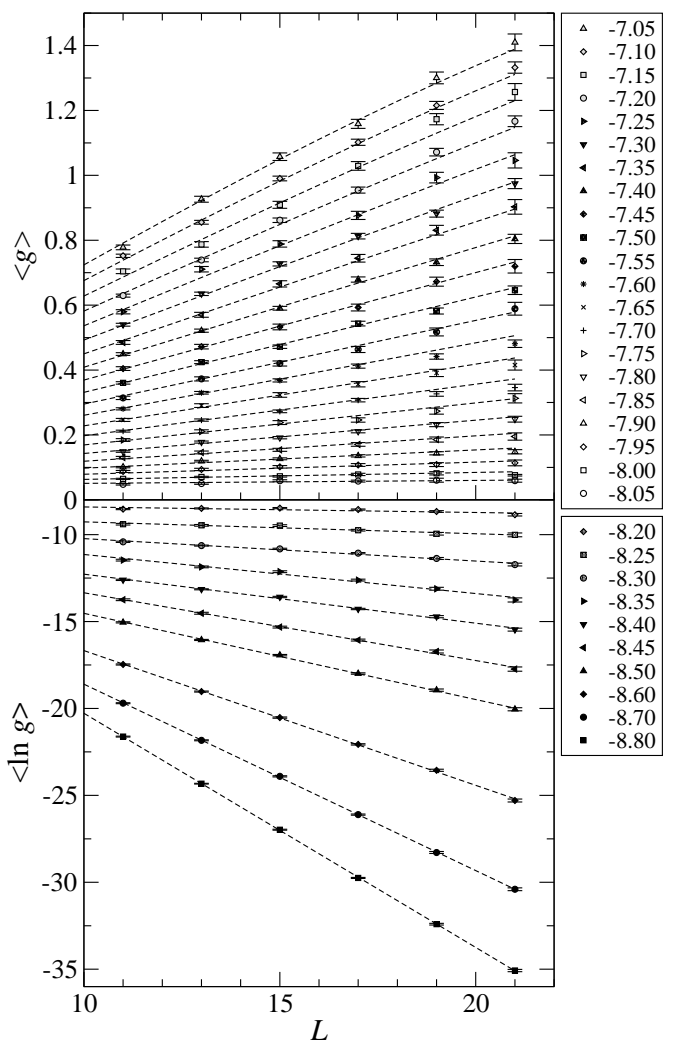

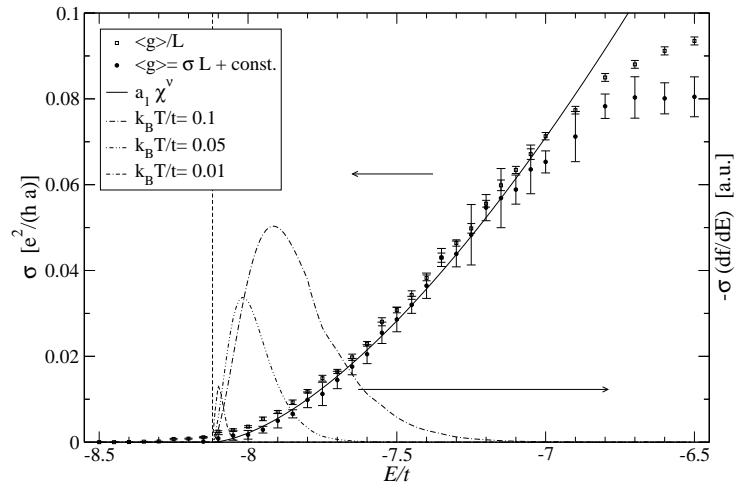

Fig. 1 (top) Conductivity $\sigma$ vs energy computed from $\langle g\rangle / L$ $(\square)$, a linear fit with $\langle g\rangle=\sigma L+$ const. $(\bullet)$ and a scaling according to Eq. (7) (solid line). The dashed line indicates $E_{\mathrm{c}} / t=-8.12$. Error bars of $\langle g\rangle / L$ represent the error-ofmean obtained from an ensemble average. Also shown is the integrand of Eq. (4) for $i=j=1$ and three different temperatures (dash-dotted).

Fig. 2 (left) System size dependence of the 4-point conductance averages $\langle g\rangle$ and $\langle\ln g\rangle$ for $W / t=12$ and Fermi energies are given in the legend. Error bars obtained from ensemble average. The dashed lines indicate the fit results to Eq. (7) and a linear function to $\langle g\rangle$ and $\langle\ln g\rangle$, respectively.

system size $L$ and the constant of proportionality is negative. This corresponds to an exponential decay of the conductance with increasing $L$ and is characteristic for insulating behaviour. For $E_{\mathrm{F}} / t$ being larger than $-8.05,\langle g\rangle$ is proportional to $L$. This indicates the metallic regime.

Following Ref. [14] we fit the data in the respective regimes to a standard scaling form, i.e. we assume

$$
\langle g\rangle=F\left(\chi L^{1 / \nu}\right) \quad \text { and } \quad\langle\ln g\rangle=F\left(\chi L^{1 / \nu}\right),
$$

where $\chi$ is a relevant scaling variable. The results for the critical exponent and the mobility edge are given in Table 1 The obtained values for both types of averages, $\langle g\rangle$ and $\langle\ln g\rangle$, are consistent. The average value of $\nu=1.59 \pm 0.18$ is in accordance with results for conductance scaling at $E_{\mathrm{F}} / t=0.5$ and transfer-matrix calculations [5, 14].

In order to obtain the anticipated power-law form for the conductivity $\sigma(E)$ in the critical regime, we need to assume the following scaling law for the conductance,

$$
\langle g\rangle=f\left(\chi^{\nu} L\right) .
$$

Then we expand $f$ as a Taylor series up to order $n_{\mathrm{R}}$ and $\chi$ in terms of the parameter $\varepsilon=\left(E_{\mathrm{c}}-E\right) / E_{\mathrm{c}}$ up to order $m_{\mathrm{R}}$. This procedure gives

$$
f\left(\chi^{\nu} L\right)=\sum_{m=0}^{n_{\mathrm{R}}} a_{m}\left(\chi^{\nu} L\right)^{m} \quad \text { and } \quad \chi(\varepsilon)=\sum_{n=1}^{m_{\mathrm{R}}} b_{n} \varepsilon^{n} .
$$

The best fit is determined by minimising the $\chi^{2}$ statistic. Using $n_{\mathrm{R}}=3$ and $m_{\mathrm{R}}=2$ we obtain for the critical values, $\nu=1.58 \pm 0.18$ and $E_{\mathrm{c}} / t=-8.12 \pm 0.03$. These values are consistent with our previous fits. The $m=1$ term $a_{1} \chi^{\nu}$ in $f$ corresponds to the conductivity close to the MIT. To estimate the quality 


\begin{tabular}{lcccccc} 
average & $E_{\min } / t$ & $E_{\max } / t$ & $n_{\mathrm{R}}$ & $m_{\mathrm{R}}$ & $\nu$ & $E_{\mathrm{c}} / t$ \\
\hline \hline$\langle g\rangle$ & -8.2 & -7.4 & 3 & 2 & $1.60 \pm 0.18$ & $-8.14 \pm 0.02$ \\
$\langle\ln g\rangle$ & -8.8 & -7.85 & 3 & 2 & $1.58 \pm 0.06$ & $-8.185 \pm 0.012$
\end{tabular}

Table 1 Best fit estimates of the critical exponent and the mobility edge for both averages of $g$ using Eq. (6). The system sizes used were in both cases $L=11,13,15,17,19,21$.

of this procedure we calculate the conductivity from the slope of a linear fit to $\langle g\rangle$ throughout the metallic regime, and also from the ratio $\langle g\rangle / L$. The resulting conductivities are shown in Fig. 1]

5 Thermoelectric Transport Knowing the energy dependence of $\sigma$ it is straightforward to evaluate Eq. (4) and to compute the temperature dependence of $\sigma$ and $S$ using Eq. (3). To investigate the consequences of deviations from the power-law behaviour of $\sigma(E)$ on $S\left(T, E_{\mathrm{F}}\right)$ we only use $\sigma=a_{1} \chi^{\nu}$ for energies close to the MIT $\left(E_{\mathrm{F}} / t \leq-7.8\right)$. Otherwise an interpolation to $\sigma$ obtained from the numerical data is used. The results for the thermopower together with the low- and high-temperature expansions using only Eq. (1) are shown in Fig. 3] Deviations from the pure power-law result [15] accumulate above $k_{\mathrm{B}} T / t \approx 0.5$.

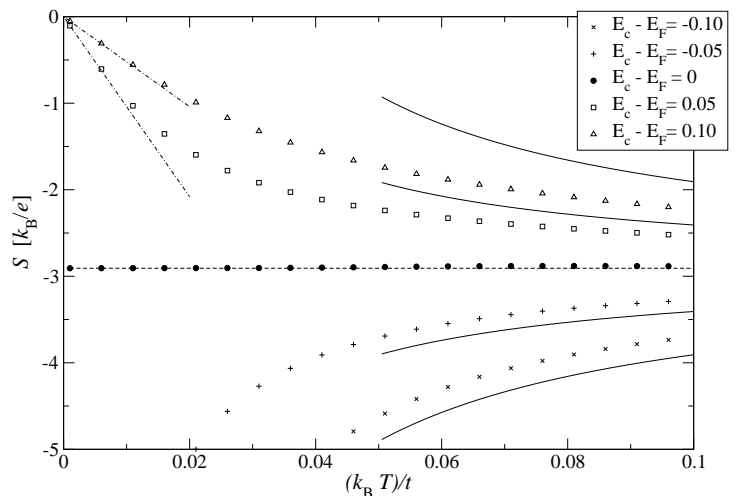

Fig. 3 Thermopower $S$ vs a dimensionless temperature parameter $k_{\mathrm{B}} T / t$. Empty symbols indicate metallic, filled symbols critical and stars insulating behaviour. The distance from $E_{\mathrm{c}}$ is given in the legend. Full lines are obtained from a high-temperature expansion [15]. The dashed lines indicate $S$ using the Sommerfeld expansion [15]. In both cases $\nu$ and $E_{\mathrm{c}}$ obtained from the fit to Eq. (7) are used.

In Fig. 1)one can see that at this temperature the integrand in Eq. (4) has already a significant contribution from the pure numerical data. Nevertheless, the deviations of $S$ from the expansions are small even for higher temperatures. This indicates a certain robustness of the results in Ref. [15].

Acknowledgements We thank C. Sohrmann and M. Schreiber for useful discussions.

\section{References}

[1] P. W. Anderson, Phys. Rev. 109, 1492 (1958).

[2] B. Kramer and A. MacKinnon, Rep. Prog. Phys. 56, 1469 (1993),

[3] E. Abrahams, P. W. Anderson, D. C. Licciardello, and T. V. Ramakrishnan, Phys. Rev. Lett. 42, 673 (1979).

[4] P. A. Lee and T. V. Ramakrishnan, Rev. Mod. Phys. 57, 287 (1985).

[5] K. Slevin and T. Ohtsuki, Phys. Rev. Lett. 82, 382 (1999); T. Ohtsuki, K. Slevin, and T. Kawarabayashi, Ann. Phys. (Leipzig) 8, 655 (1999); R. A. Römer and M. Schreiber, in The Anderson Transition and its Ramifications - Localisation, Quantum Interference, and Interactions, edited by T. Brandes and S. Kettemann (Springer, Berlin, 2003), pp. 3-19.

[6] F. Wegner, Z. Phys. B 25, 327 (1976).

[7] A. MacKinnon, J. Phys.: Condens. Matter 13, L1031 (1980). —, Z. Phys. B 59, 385 (1985).

[8] H. B. Callen, Thermodynamics and an Introduction to Thermostatistics (John Wiley \& Sons, New York, 1985).

[9] G. V. Chester and A. Thellung, Proc. Phys. Soc. 77, 1005 (1961).

[10] D. A. Greenwood, Proc. Phys. Soc. 71, 585 (1958); R. Kubo, J. Phys. Soc. Japan 12, 570 (1957).

[11] B. K. Nikolić, Phys. Rev. B 64, 165303 (2001).

[12] R. Landauer, Phil. Mag. 21, 863 (1970); M. Büttiker, Phys. Rev. Lett. 57, 1761 (1986).

[13] M. Büttiker, Phys. Rev. B 38, 9375 (1988).

[14] K. Slevin, P. Markoš, and T. Ohtsuki, Phys. Rev. Lett. 86, 3594 (2001).

[15] C. Villagonzalo, R. A. Römer, and M. Schreiber, Eur. Phys. J. B 12, 179 (1999). 